\title{
Douleur : quel monitorage pour quel patient ?*
}

\section{Pain: Which Kind of Monitoring for Which Patient?}

\author{
D. Charier $\cdot$ S. Molliex
}

(C) Lavoisier SAS 2017

Un stimulus nociceptif entraîne une réponse adaptative de l'organisme, conduisant le plus souvent à une activation du système sympathique. L'enregistrement du système nerveux autonome (SNA) permet ainsi une évaluation de la douleur chez le sujet éveillé ou de la nociception chez le patient sous anesthésie générale.

Le principal objectif de ces techniques de monitorage du SNA est de permettre une utilisation plus rationnelle des antalgiques chez les patients non communicants (âges extrêmes de la vie, pathologie neurologique ou psychiatrique, réveil anesthésique, patients de réanimation) ou en peropératoire, évitant les sur- ou les sous-dosages en opioïdes, améliorant ainsi la stabilité hémodynamique des patients les plus fragiles, tout en minimisant les phénomènes d'hyperalgésie postopératoire.

L'analgésie se définit comme la persistance d'un état d'équilibre après un stimulus nociceptif (" résistance au changement »), la douleur ou la nociception comme un déséquilibre. Pas plus qu'une valeur isolée de fréquence cardiaque ou de pression artérielle ne le permet, aucune valeur instantanée des paramètres d'enregistrement du SNA ne peut donner de mesure objective de la douleur : l'évaluation de la douleur repose nécessairement sur une mesure dynamique, comparant par exemple un « état de repos » à un « état douloureux » ou une valeur « avant traitement antalgique » et une valeur « après traitement ».

La conductance cutanée, le temps de transit du pouls ou la variabilité de la fréquence cardiaque permettent ainsi une évaluation en continu de la balance sympathique-parasympathique, reflet de la réponse du SNA à la douleur. En particulier, l'Analgesia Nociception Index ${ }^{\circledR}$ (ANI ${ }^{\circledR}$, MDoloris, Lille), paramètre dérivé de la variabilité de la fréquence car-

D. Charier $(\bowtie) \cdot$ S. Molliex

Département d'anesthésie-réanimation, hôpital Nord,

CHU de Saint-Étienne, F-42055 Saint-Étienne cedex 2, France

e-mail : david.charier@chu-st-etienne.fr

\footnotetext{
* Résumé de la communication faite le 31 mars 2017 à Lyon lors du congrès de la Société française d'évaluation et de traitement de la douleur.
}

diaque, permet une mesure continue de la composante parasympathique, avec un délai de 16 à 64 secondes.

De même, la vidéopupillométrie permet aujourd'hui une évaluation simple de la douleur chez des patients éveillés ou sous anesthésie générale. Ici encore, seuls des tests dynamiques, comme la mesure du réflexe de dilatation pupillaire à la douleur ou du réflexe photomoteur, peuvent apporter une information, a posteriori, en 250 à 750 millisecondes, sur le déséquilibre induit par une stimulation nociceptive, et donc renseigner sur l'équilibre douleur-analgésie ou nociceptionantinociception à un instant donné. De même, le monitorage des fluctuations permanentes du diamètre pupillaire, paramètre récemment décrit et implémenté dans le vidéopupillomètre AlgiScan ${ }^{\circledR}$ (iDMed, Marseille), apparaît comme une mesure dynamique susceptible d'apporter une information sur l'équilibre douleur-analgésie, avec l'avantage de pouvoir être réalisé en continu [1].

La vidéopupillométrie, comme les autres paramètres d'évaluation du SNA, peut ainsi permettre une optimisation de la prise en charge de la douleur chez des patients dont l'autoévaluation est considérée comme peu fiable ou une adaptation individuelle des besoins en antalgiques aux différents temps chirurgicaux chez des patients sous anesthésie générale.

Liens d'intérêts : Les auteurs déclarent ne pas avoir de lien d'intérêt.

\section{Référence}

1. Charier D, Zantour D, Pichot V, et al (2017) A new pupillary measure to assess pain: a prospective study. J Pain (accepted for publication) 